NOTICE: This is the author's version of a work that was accepted for publication in Journal of Nuclear Materials. Changes resulting from the publishing process, such as peer review, editing, corrections, structural formatting, and other quality control mechanisms may not be reflected in this document. Changes may have been made to this work since it was submitted for publication. A definitive version was subsequently published in Journal of Nuclear Materials, 413, 3, 2011. DOI: 10.1016/j.jnucmat.2011.04.015 


\title{
Buckle, ruck and tuck: a proposed new model for the response of graphite to neutron irradiation
}

\author{
M.I. Heggie ${ }^{1}$, I. Suarez-Martinez ${ }^{2}$, C. Davidson ${ }^{1}$, G. Haffenden ${ }^{1}$ \\ ${ }^{1}$ Chemistry Subject Group, School of Life Sciences, University of Sussex, \\ Falmer, Brighton, BN1 9QJ, UK \\ ${ }^{2}$ Nanochemistry Research Institute, Department of Chemistry, Curtin University \\ of Technology, GPO Box U1987, Perth, Western Australia 6845
}

Abstract

The default theory of radiation damage in graphite invokes Frenkel pair formation as the principal cause of physical property changes. We set out its inadequacies and present two new mechanisms that contribute to a better account for changes in dimension and stored energy. Damage depends on the substrate temperature, undergoing a change at approximately $250^{\circ} \mathrm{C}$. Below this temperature particle radiation imparts a permanent, nano-buckling to the layers. Above it, layers fold, forming what we describe as a ruck and tuck defect. We present first principles and molecular mechanics calculations of energies and structures to support these claims. Necessarily we extend the dislocation theory of layered materials. We cite good experimental evidence for these features from the literature on radiation damage in graphite.

Keywords: Graphite, nuclear, irradiation, fold, buckle, ruck, Wigner

Graphite has a 60 year history as a moderator in nuclear reactors and its radiation damage is thus a mature research field. The structures and reactions we propose here could help rationalize unsolved puzzles and quantitative inadequacies in the accepted wisdom. If they are confirmed experimentally, the improved understanding of current empirical models should bear directly on the design of next generation fission and fusion nuclear reactors and on the operation and decommissioning of current graphite moderated reactors.

Historically, the consensus had evolved that radiation-created interstitial atoms aggregate to form new graphite sheets (graphenes)(1). In the last decade or so high resolution electron microscopy (HREM) has produced striking images of graphene sheets, side on, including carbon nanotubes of single, double or multiwalled nature(2). In the analysis of graphite damaged by particle radiation (electrons, neutrons or ions), such HREM images $(3,4)$ reveal bending and breaking of sheets, but little or no evidence of the growth of new sheets from the aggregation of interstitial atoms. This is a problem for the standard (atomic displacement) model for radiation damage of graphite and the traditional explanation for dimensional changes and energy storage, which invoke point defect aggregation into new sheets. In this article we show that the standard 
model has problems and that instead, the indirect evidence points to the importance of buckling and folding of sheets. A speculation by Jenkins(5) anticipates the possible importance of buckling, at least for large doses.

It is not unreasonable to suppose that such buckling and folding could be intrinsic to all layered materials, albeit in varying degrees dependent on the rigidity of the layers and the strength of the interlayer interactions.

There has been a very recent wide-ranging review of radiation damage in graphite (6) to which we refer the reader. Figure 1(a) contains some essential features. It is a schematic of dimensional changes in highly oriented pyrolytic graphite (HOPG) as a function of neutron dose at different temperatures. Fractional dimensional changes parallel to c, i.e. $\Delta X_{c} X_{c}$, are linear to parabolic and do not saturate with dose above $250^{\circ} \mathrm{C}$. Above this temperature they are sigmoidal, i.e. eventually saturate. The lower dashed curves, perpendicular to $\boldsymbol{c}$, i.e. $\Delta X_{a} / X_{a}$, show basal plane contraction, originally thought to be due to the aggregation of vacancies into lines which heal and contract basal dimensions(1).

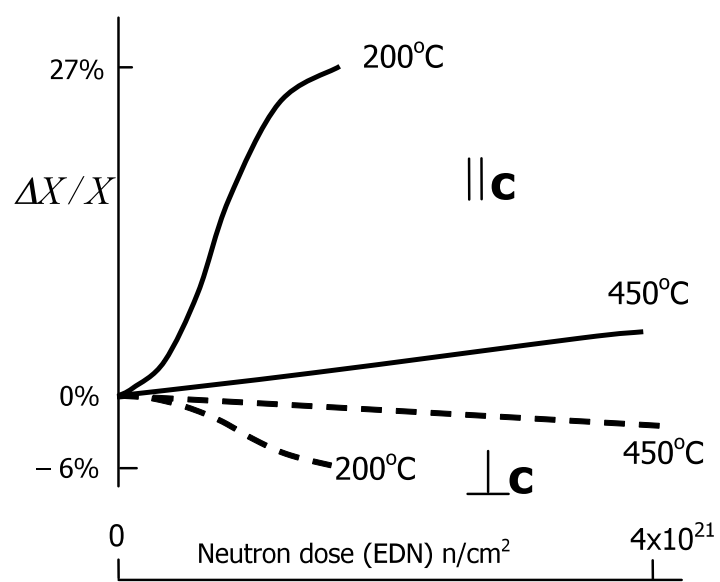

Figure 1(a): Schematic of fractional changes in dimensions $(\Delta X / X)$ parallel to $\mathrm{c}$ (solid lines,

$X=X_{c}$ ) and perpendicular to $\mathrm{c}$ (broken line, $X=X_{a}$ ) in HOPG as a function of neutron dose(7)

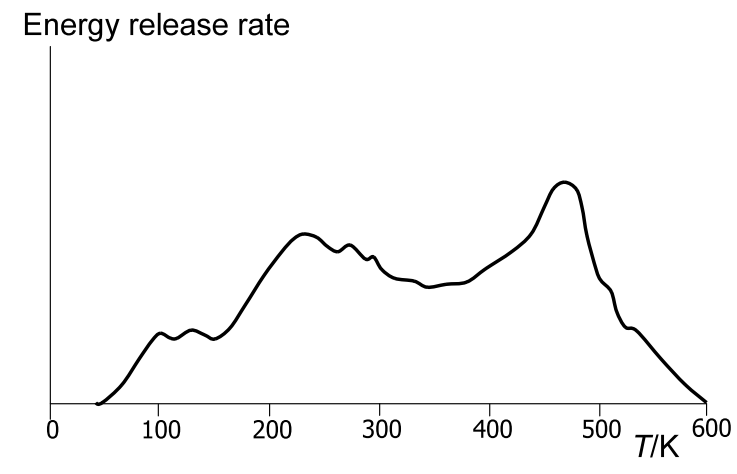

Figure $1(b)$ Annealing of radiation damage performed at $20 \mathrm{~K}(7,8)$

Turning to the storage of energy, this occurs during radiation damage mainly at temperatures below $250^{\circ} \mathrm{C}$. The release of stored (or Wigner) energy from irradiated graphite is normally monitored by ramping the temperature linearly with time. The resulting energy release as a function of temperature is generally interpreted as a set of energy traps releasing in sequence as their different release activation energies become accessible with the rising temperature. For cryogenic irradiations, figure 1(b), energy release starts at liquid nitrogen temperature (77K). Wigner energy is thought to be largely in the form of point defect formation energies which release as point defects aggregate or annihilate. The pronounced $200^{\circ} \mathrm{C}$ peak can release more heat than the heat capacity of the 
graphite, and so cause runaway temperature rises. It has been identified as the cause of the 1957 accident in Windscale Pile 1(9), although we note that current operational reactors operate well above $250^{\circ} \mathrm{C}$ and are not susceptible to it. One of the first quantitative applications of our DFT technique to this problem (10) showed that the annihilation of the intimate Frenkel pair with activation energy 1.3-1.4 eV could be an important contribution to this peak, and subsequent HREM studies of interwall defects in double walled nanotubes (2) appear to reinforce this finding.

Aggregation of interstitials causes prismatic dislocation loops which have long been seen in weak beam transmission electron microscopy (TEM) (11) and thus invoked as key to the radiation damage process $(1,12)$. The migration energy of the interstitial can be deduced to be ca. $1.2 \mathrm{eV}$ from the activation energy of aggregation into interstitial loops $(13,14)$. We have confirmed this value is at least a lower bound directly by saddle point location using DFT calculation. Movement of interstitials at $77 \mathrm{~K}$ is thus ruled out and the standard model cannot explain the data of figure 1(b). Even if the interstitial could be sufficiently mobile, the amounts of dimensional change arising from the prismatic loops of the standard model are reported as less than a half, and most often a tenth, of the measured changes (14).

We also rule out the possibility that small defect clusters can substantially change interlayer spacings, as has been invoked in the interpretation of small angle cold neutron scattering measurements (15). None of the defect cluster structures we have studied can realistically account for the large differential dimensional changes seen at, for instance, $200^{\circ} \mathrm{C}$ in figure $1(\mathrm{a})$. Nor can the cluster formation be easily reversible, since dimer formation is exothermic by 3 eV (16).

Finally in summarising experimental evidence we note X-ray diffraction experiments on neutron irradiated natural graphite, using both oscillating and precession X-ray diffraction techniques. These show two distinct features: one is consistent with rods passing through all $h k i 0$ reflexions (17) and the other, diffuse streaks along all $h k$ rel-lines (18). The only satisfactory explanation of the first is translation of layers by random amounts in the [11이 direction (but this was rejected because there was no known mechanism to generate them) and the likely explanation for the second is the appearance and growth of line defects. The formation and movement of basal dislocations consequent upon neutron irradiation are well documented for low doses (11). Basal dislocations cause basal plane shifts and are line defects. They are thus the most obvious and economical explanation for both these diffraction features and for physical property changes as we shall show below.

First we rehearse and refine our preliminary proposal of graphene buckling on a nanometer scale (19) as the principal structural change in graphite upon 
irradiation below $250^{\circ} \mathrm{C}$. This is the only model of radiation damage to date that is motivated by first principles calculation in atomistic detail. We note that there has been some speculation about buckling, and in particular of the circumstance where the cores of non-basal edge dislocations, i.e. $\underline{l}=[0001], \underline{b}=[1-100]$ give rise to interlayer bonds(5). These dislocations, whose structure and movement has been analysed by one of us with first principles calculations(20), are completely different in character from basal dislocations and their formation is much hindered by requiring the breaking of $\mathrm{C}-\mathrm{C}$ bonds. It does not seem likely that this form of interlayer pinning will dominate over the point-defect based mechanisms proposed here.

Then we move on to a further proposal (21), that higher temperature damage is characterized by folding of the layers (and the formation of the ruck and tuck defect). It appears that historical models were principally informed by ideas of a two dimensional gas of point defects condensing in different ways, with the graphite layers providing a more or less rigid framework within which motion occurred. Here we add a new concept - deformation of the layers - and we provide a way of understanding this deformation through innovations in dislocation theory of layered materials, invoking observable defects (basal dislocations).

Turning first to the new model for irradiation damage below $250^{\circ} \mathrm{C}$, the most remarkable and almost universal feature of point defects that we have studied with DFT, i.e. the spiro-interstitial, di-interstitial, interplanar divacancy and intimate Frenkel pair, is the formation of strong interlayer bonds (22) and this is the key evidence in understanding the role of basal dislocations and buckling.

Whenever one sheet is linked in two places to another of different length, the result is buckling - compression buckling of the longer sheet is easier than stretching of the shorter, which tends to stay close to its unstrained length. The two graphene sheets either side of the glide plane in a basal dislocation differ in length by the edge component of the Burgers vector. The core of a basal dislocation in a simple model is approximately $4 \mathrm{~nm}(23)$. It is uncertain if buckling can occur for an isolated dislocation. However, when the core is artificially localized by pinning points or when the dislocation interacts with another of opposite sign in a nearby interplanar region, buckling can occur.

This is clearly illustrated in the supercell of figure 2(a), of original dimensions $|c|$, $10|a|$, and $\sqrt{ } 3|a|$, i.e. $0.67 \mathrm{~nm}, 2.46 \mathrm{~nm}, 0.426 \mathrm{~nm}$. Removing a lattice vector, $|a|$, of material from one sheet shortens it to $9|a|$ and produces an average supercell width of $91 / 2|a|$. Optimization of geometry and superlattice vectors shows that the buckling attains the highest wavelength possible (the supercell width) and the dimensional changes are $\Delta X_{a} / X_{a}=-5 \%$ and $\Delta X_{d} / X_{c}=63 \%$, i.e. buckling is clearly capable of reproducing curves of Figure 1(a). While being a simple and appealing two dimensional model of graphite damaged below $250^{\circ} \mathrm{C}$, it is striking also as a new material, featuring buckled sheets. A simplified generalisation to 
three dimensions invokes buckling along different directions in different planes and more generally, crumpled sheets where the direction of buckling varies within the same sheet, but the two dimensional model captures most of the physics, and applies where the length of the defect greatly exceeds its width.

Dislocation theory offers the most compact and helpful description of this structure of figure 2(a). It is lattice of supercells each containing a climb dipole of perfect basal edge dislocations (of axes, $\boldsymbol{I}=<1 \overline{1} 00>$, Burgers vector, $\boldsymbol{b}=1 / 3 \boldsymbol{a}$ $<11 \overline{2} 0>$ and separation $1 / 2 \quad c<0001>$ ). Climb dipoles do not glide on the same plane. If they were to glide on the same plane (i.e. the interlayer space) they would annihilate, restoring graphite structure and releasing energy. However in our structure the two infinite half-planes which arise from the edge component collapse to form a complete crystal plane and a column of extra material (which is the cause of the buckling). Typically, if two perfect basal edge dislocations separated by one micrometer collide to make a climb dipole, the elastic energy stored in their strain fields (which is of the order of $10^{3} \mathrm{eV} / \mathrm{nm}^{-}{ }^{-}$) is lost, providing more than enough energy to fuel the buckling process above and hence dimensional change.

A finite macroscopic crystal of this kind cannot be stable with respect to unbuckling. A structure which should be metastable to $200^{\circ} \mathrm{C}$, is shown in Figure 2(b). Here dislocations glide together on different planes, causing buckling and are pinned in place by interplanar bonds (in this case from spiro-interstitials). The dimensional change is $\Delta X_{a} / X_{a}=-4 \%, \Delta X_{c} / X_{c}=48 \%$, also compatible with that achieved in experiment below $250^{\circ} \mathrm{C}$.

Interlayer defects can resist a certain (critical) shear stress before yielding and allowing the dislocation to slip past (each dislocation passage shears one plane past the other by its Burgers vector, i.e. a basal lattice vector in the case of a perfect dislocation). As the temperature increases the critical shear stress reduces by thermally activated jumping, decreasing to zero by $250^{\circ} \mathrm{C}$, when the majority of the interlayer defects either annihilate or migrate rapidly. The buckling therefore disappears at this temperature.

The amount of energy stored is $16.5 \mathrm{eV}$ in the unit cell of Figure 2(b). Approximately $2 \times 5.7 \mathrm{eV}$ should come from the two undistorted interstitials and the rest, $5.1 \mathrm{eV}$, from buckling and internal stresses - i.e. nearly one third of the energy stored in this cell is in the buckling. Modern Wigner energy measurements (24) show that $90 \%$ of stored energy is released by $300^{\circ} \mathrm{C}-$ we argue this is largely from a combination of intimate Frenkel pair annihilation, buckling removal and point defect migration and aggregation. 

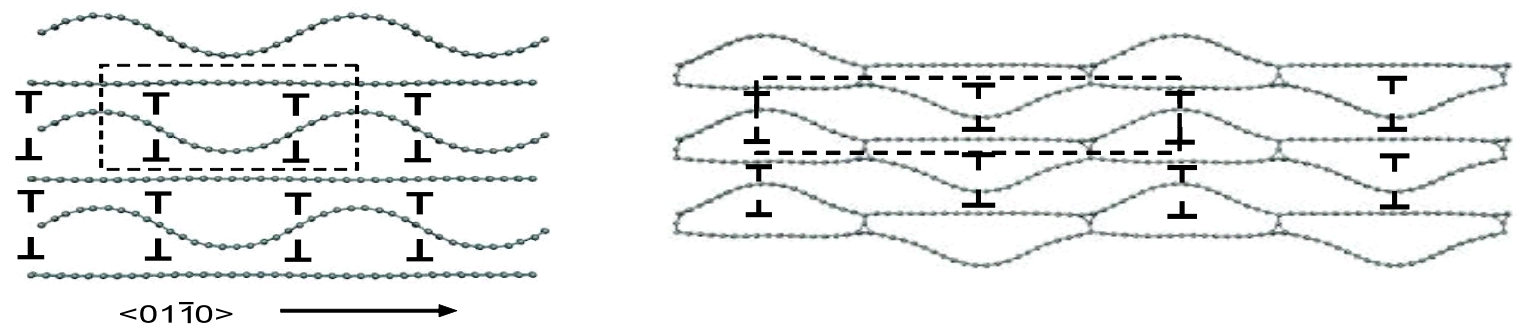

Figure 2 Buckled graphite (DFT optimized structures). Dislocations are represented by the T symbol and the supercell is depicted by broken lines.

(a) Superlattice of climb dipoles of basal edge dislocations

(b) Buckled graphite held by interlayer pinning points (in this case, spiro-interstitials).

A recent molecular dynamics simulation of damage from Ar ions has appeared (25) and it demonstrates swelling from two neighbouring spiro interstitials. This can be viewed as a point- (rather than line-) defect version of Fig. $2 \mathrm{~b}$. It might also be regarded as confinement of a basal dislocation core, which otherwise would have a width of some $4 \mathrm{~nm}(23)$.

Before moving on to the rucking of planes, we note a compelling argument supporting the buckling model. Electrical resistivity parallel to the planes increases, and perpendicular to the planes decreases, with irradiation dose. The former is due to electron scattering by defects disrupting in-plane bonding and the latter is due to the extra coupling between layers from the interlayer bonds (22). A longstanding enigma has been that annealing cryogenic (liquid $\mathrm{Ne}$ or $\mathrm{He}$ ) radiation damage does not restore resistivity monotonically to its original values, but rather causes the radiation-induced trends to proceed in the same direction, as if radiation were continuing, in the early stages of annealing $(26,27)$. There have been attempts to interpret this phenomenon as initial aggregation of interstitials into small clusters which can break up as the temperature is raised. For this break up to occur around $100 \mathrm{~K}$ would seem to imply and extraordinarily fine balance of free energies between these small clusters and isolated interstitials. Contrarily, the formation of interstitial dimers has been shown to be highly exothermic (16) and largely irreversible. The rather successful quantitative model of the kinetics of loop growth is predicated on irreversible formation of $\mathrm{C}_{2}$ units as the first nucleation step (13).

A straightforward interpretation of this reverse annealing phenomenon is that planes may become so buckled that interlayer defects cannot form. Instead, interstitials, for example, can only be adatoms associated with one plane (Figure $3 a)$. In the first annealing stages the unbuckling allows their conversion into interlayer defects, giving all the appearance of continued irradiation (Figure 3b).
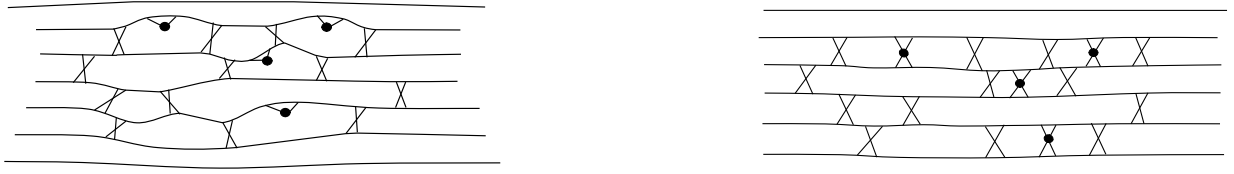
Figure 3(a) Schematic post cryo-irradiation. Buckling caused by spiro-interstitials (represented by crosses). Severe buckling leaves adatoms unable to cross-link (black circles).
Figure 3(b) After a short anneal 'new' interstitials, i.e. cross-linking defects, appear (crosses with black circles).

Having put forward the case for the formation of buckled graphite in irradiation below $250^{\circ} \mathrm{C}$, we turn to the folded or ruck-and-tuck graphite (21) which we suggest arises at higher temperatures.

Whereas at low temperatures basal dislocations are pinned by interlayer defects, at temperatures higher than $250^{\circ} \mathrm{C}$ most such pinning points disappear $(2,10)$. The mean free path of basal dislocations increases unfettered and they are free to expand and mutually interact more strongly. Thus the encounters between dislocations of opposite sign on different glide planes can be multiple. A 'pile up' can contain many Burgers vectors as illustrated in figure 4, where four perfect basal edge dislocations have piled up. Conventionally, there is an accumulation of matter at the crossing and annihilation of these four dislocations. In layered materials, the provenance of this matter is important: what belonged to one layer, still belongs to that layer. The consequence is a fold (or ruck and tuck) (figure $4(a))$

As an aside, it appears ironic that a ruck in a carpet is part of the pedagogical imagery for the role of dislocations in facilitating plastic deformation in all materials(28), yet has eluded discussion in the case of layered materials materials which are most like stacks of carpets.

Figure 4 illustrates the process for two sets of four basal edge dislocations which are forced by neutron collisions to pile up and glide past each other on nearby planes. The result is a 'ruck and tuck' defect (shown schematically in 4(a)), which has two alternate descriptions in dislocation theory. In addition to being considered as a pile up of climb dipoles (Figure 4(b), where the dislocations are $\boldsymbol{b}=1 / 3$ a $\langle 11 \overline{2} 0>, \boldsymbol{I}=\langle 1 \overline{1} 00>$ ), it can also be regarded as an unfaulted climb dipole (Figure 4(c), where the dislocations are $\boldsymbol{b}=c<0001>, \boldsymbol{I}=<1 \uparrow 00>$ ).

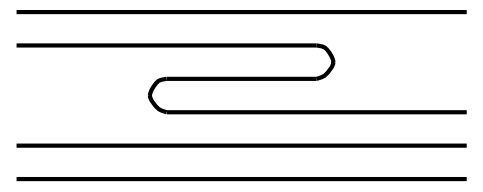

Figure 4(a) Ruck and tuck defect (schematic). The relaxation of the planes is not shown.

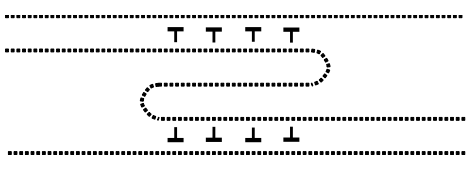

Figure 4(b) Pile up of basal edge climb dipoles. T symbols are dislocations, graphene planes are dotted.

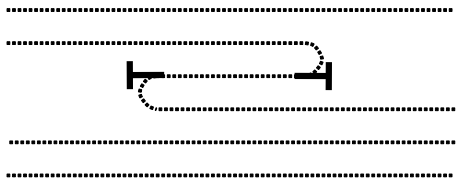

Figure 4(c) Unfaulted climb dipole of prismatic dislocations. T symbols are dislocations, graphene planes are dotted. 
Thus, formation of a prismatic climb dipole, which is close in nature to the loops of the standard model and is normally regarded as requiring climb via point defect migration, can be achieved by basal glide alone. This is a very important point: in a layered material the glide of basal edge dislocations can perform mass transport.

The existing model for $\mathrm{c}$ axis expansion confines itself to Frenkel pair formation and relies on the migration of interstitial atoms to disks of one layer thickness, which may or may not be sheared. Such disks are bounded by a loop of prismatic edge dislocation. These dislocations are not 'perfect' but are 'partial' since their Burgers vectors $(\underline{\mathrm{c}} / 2)$ are not lattice vectors. The model satisfies two key observations for dimensional change above $250 \mathrm{C}$ : the interlayer separation by $X$ ray diffraction, $d_{002}$, is maintained at approximately $0.34 \mathrm{~nm}$ and the $c$ axis dimension expands without apparent limit. As discussed earlier, there had been a difficulty in understanding the kinetics of growth of such disks, since the activation energy for nucleation and growth was measured to be about $1.2 \mathrm{eV}$, much greater than the then accepted range of values for interstitial migration (0.04-0.4 eV (29). In the light of modern first principles calculations revealing that the interstitial is bonded, it can now be more easily accepted that $1.2 \mathrm{eV}$ can be the effective migration energy of the interstitial in the basal plane.

The model presented here allows for the formation of columns of extra material two layers thick, corresponding to a dipole of perfect prismatic edge dislocations. The extra material has arisen uniquely from glide of basal edge dislocations. It is as though moving basal dislocations achieve mass transport by depositing part of their 'extra half plane'.

Returning to the issue of the $c$ axis dimension appearing to expand without limit, if we were to consider the limiting case of a highly dense parallel system of ruck and tuck defects separated by one interlayer separation in the c direction and, say 10 micrometer in the a direction, then the potential dimensional change of a macroscopic piece of graphite $1 \mathrm{~cm}$ across, is $\delta X_{c} / X_{c}=1,000$. Thus it does not seem impossible that dimensional change will appear continuous and nonsaturating.

The ruck and tuck formation process can be much more easily reversible than diffusive mechanisms. At very high damage temperatures $\left(>800^{\circ} \mathrm{C}\right) 60-80 \%$ of the dimensional change is unannealable (30). Energy calculations for point defect aggregation, which is at the heart of the standard model, are always extremely endothermic making it effectively irreversible under all but extreme temperatures, so high temperature damage must involve a substantial role for the standard model. Damage at lower temperatures is, contrarily, almost completely annealable in pyrolytic carbons (30), and thus incompatible with the standard model and indicative of the dominance of the new model, where annealing can allow the pile up process to reverse. 
We note that the single dislocation dipole, especially if it has a multiple Burgers vector, could restructure into the 'ruck and tuck' defect of Figure 4(a), but it can also be created by the sequential collisions of two or more oppositely signed basal edge dislocations gliding towards each other on nearby planes.

This defect could also arise by a progressive pile up of basal dislocations. Pile up is normally resisted by the Peach Köhler force between dislocations of like-sign, but in graphite this force is strongly diminished by elastic anisotropy and the ease of accommodating material through expansion perpendicular to the planes. Figure 5 illustrates how passage of a single basal dislocation can grow the prismatic dipole. It does so by 'depositing' a segment of its extra half plane at the ruck and tuck defect. Two edge dislocations of opposite sign moving in opposite directions on neighbouring glide planes can nucleate one of these defects. Note that compression along $\boldsymbol{c}$ will suppress this climbing effect, tension along $\boldsymbol{c}$ will enhance it. Pairs gliding on more distant planes can in principle give multilayer folds (for brevity not considered here) and basal dislocations can dissociate into partials, so ruck and tuck defects do not necessarily comprise integral numbers of basal lattice vectors. We adopt the chiral index notation of carbon nanotubes to label the defects, $(j, k)$ giving the length and direction of the folded material, and add a third index, $n$, for the number of layers folded. In the 'perfect' case, these are all integers, the two folds are parallel to each other and perpendicular to the chiral vector. The length of each added sheet is $l=a \sqrt{j^{2}+j k+k^{2}}$.

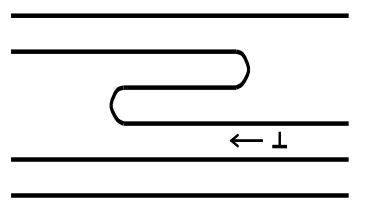

(a)

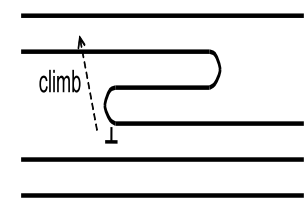

(b)

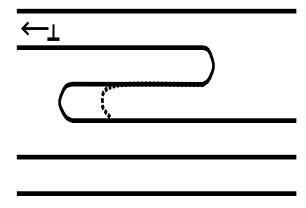

(c)

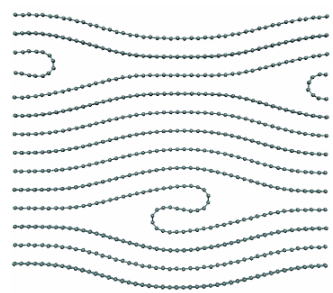

(d)

Figure 5 (a-c) A basal edge dislocation sweeping right to left (a), climbs a plane (b) and extends the ruck and tuck defect (c). Figure 5(d) Orthorhombic superlattice of two $(10,0) \times 1$ ruck and tuck defects. There are $80 \mathrm{C}$ added in two sets of $40 \mathrm{C}$ in a unit cell which initially has $1104 \mathrm{C}$

Figure $5(\mathrm{~d})$ is an example of a pair of $(10,0) \times 1$ ruck and tuck defects (31) optimized by the Universal Force Field (32) implemented in Cerius ${ }^{2}{ }_{\circledast}$ which has been found to give principal elastic constants close to those of DFT/LDA calculations. There are 80 added atoms, equivalent to two $(10,0)$ nanotubes, and the resulting radii of curvature are between 0.22 and $0.28 \mathrm{~nm}$, i.e. curvature equivalent to small zigzag nanotubes of index $(6,0)$ to $(7,0)$. The dimensional change from a comparable perfect graphite cell is $\Delta X_{a} \mid X_{a}=-9 \%, \Delta X_{c} / X_{c}=18 \%$. If the line density of ruck and tuck defects of type $(j, k) \times n$ is $p$ (line length per unit area) then the fractional $c$ dimensional change arising from purely geometric considerations in the large $l$ limit is $l p n d_{002}$ where $d_{002}$ is the interlayer spacing. In 
this case $p=9 \times 10^{12} \mathrm{~cm}^{-2}$. giving theoretical $\Delta X_{a} / X_{a}=-6 \%, \Delta X_{c} / X_{c}=14 \%$; the discrepancy is due to bending of planes and finite size effects.

The amount of energy stored per atom of the ruck and tuck is $0.46 \mathrm{eV}$ and the dimensional change it causes is close to volume conserving, as is found experimentally. Note that the atoms in the ruck arise from basal dislocation glide, not from Frenkel pairs.

Figure 6 shows diffraction pattern simulations under conditions comparable to those of Eeles experiments $(17,18)$, which relate to a nominal $200^{\circ} \mathrm{C}$ neutron irradiation of Ticonderoga flake, i.e. close to the transition between buckling and ruck and tuck, and we use the latter for simulation to account for heating effects.

Figure $6 \mathrm{a}$ Simulated oscillation Figure $6 \mathrm{~b}$ Simulated precession diffraction pattern from $(15,0) \times 1$ ruck diffraction pattern from $(8,0) \times 1$ ruck and tuck (c.f. figure 3 of ref (17), and tuck (c.f. the figure of ref (18), reproduced as Fig $6 \mathrm{c}$ )

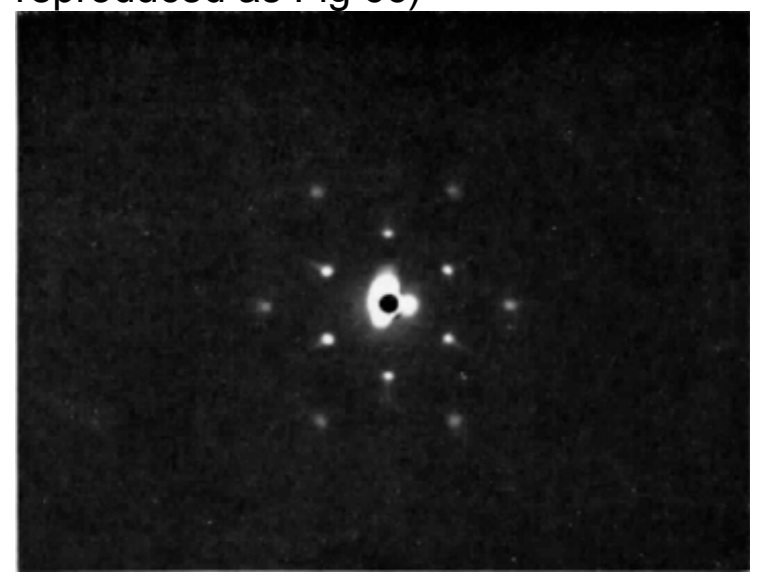
reproduced as Fig 6d)

\section{Figure 6c Figure 3 of ref (17) Figure $6 \mathrm{~d}$ The figure from ref (18) reproduced with permission from Acta reproduced with permission from Acta Crystallographica) Crystallographica)}

Given the highly individual nature of graphite samples ("no two graphite samples have ever given identical X-ray patterns in every detail" (33)) and the simplicity of the model, the agreement is very good, reproducing the relative intensities of 
inner and outer spots in Fig. 6a, and the diffuse streaks along 10 rel-lines in Figure 6b.

One final point concerns the different assumptions of this model and the standard one. The mean energy imparted by a reactor neutron to the nucleus of the primary knock-on atom (PKA) is $50 \mathrm{keV}$, increasing to $300 \mathrm{keV}$ for a head-on collision (6), which implies an instantaneous PKA speed of $\mathrm{O}\left(10^{6}\right) \mathrm{ms}^{-1}$ and an accompanying momentum. In the standard model, the energy is dissipated by further collisions, which create Frenkel pairs and heat (random atomic motion). We argue that the crystal anisotropy in graphite means that the momentum transfer retains some order, layers moving past other layers through the motion basal dislocations. We note that moving basal dislocations are able to carry momentum $(34,35)$, behaving like relativistic objects, limited not by the speed of light but by the speed of shear waves in the solid.

It is clear that acceptance of a new model will require more experimental evidence, and that application of modern electron microscopy (high resolution in a basal direction, diffraction contrast close to prismatic direction) and diffraction techniques (X-ray and neutron) to examine basal dislocations and related defects invoked here will be important in affirming or refuting the model.

In summary, we have presented two new linear structures for layered materials and given a novel and compact description in terms of dislocation theory. In the case of graphite, for which superb data exists for historical reasons, the case for these structures is strong in their two different temperature regimes. The likelihood is that most layered materials could be engineered to produce such structures with consequent novel properties. Important examples are few-layered graphene, clays, ceramic semiconductors, $\mathrm{MgB}_{2}$ and $h$-BN

Methods:

The AIMPRO code $(36,37)$ has been used with a pdpp basis (four Gaussians per atom, each combined with an expansion up to $l=1$ in spherical harmonics (an $l=2$ function is added to one of the exponents)) and $\mathrm{HGH}$ pseudopotentials (38). Special $k$ points given by Monkhorst-Pack algorithm (39).

Acknowledgments

We gratefully acknowledge support from British Energy Generation Ltd (now EDF Energy) and discussions with Mark Bradford and Alan Steer. We are grateful to Patrick Briddon for provision of AIMPRO and Gianluca Savini for computational assistance. The views expressed in this paper are those of the authors and do not necessarily represent the views of EDF Energy.

Endnotes -none

Tables -none 
Figure Legends

Figure 1(a): Schematic of fractional changes in dimensions $(\triangle X / X)$ parallel to $\mathrm{c}$ (solid lines, $X=X_{c}$ ) and perpendicular to $c$ (broken line, $X=X_{a}$ ) in HOPG as a function of neutron dose(7)

Figure 1(b) Annealing of radiation damage performed at $20 \mathrm{~K}(7,8)$

Figure 2 Buckled graphite (DFT optimized structures). Dislocations are represented by the T symbol and the supercell is depicted by broken lines.

Figure 2(a) Superlattice of climb dipoles of basal edge dislocations

Figure 2(b) Buckled graphite held by interlayer pinning points (in this case, spirointerstitials).

Figure 3(a) Schematic post cryo-irradiation. Buckling caused by spiro-interstitials (represented by crosses). Severe buckling leaves adatoms unable to cross-link (black circles).

Figure 3(b) After short anneal 'new' interstitials, i.e. cross-linking, defects appear (crosses with black circles).

Figure 4(a) Ruck and tuck defect (schematic). The relaxation of the planes is not shown.

Figure 4(b) Pile up of basal edge climb dipoles. T symbols are dislocations, graphene planes are dotted

Figure 4(c) Unfaulted climb dipole of prismatic dislocations. T symbols are dislocations, graphene planes are dotted.

Figure 5 (a-c) A basal edge dislocation sweeping right to left (a), climbs a plane (b) and extends the ruck and tuck defect (c). Figure 5(d) Orthorhombic superlattice of two $(10,0) \times 1$ ruck and tuck defects. There are $80 \mathrm{C}$ added in two sets of $40 \mathrm{C}$ in unit cell which initially has $1104 \mathrm{C}$

Figure 6a Simulated oscillation diffraction pattern from $(15,0) \times 1$ ruck and tuck (c.f. figure 3 of ref (17) reproduced as Fig 6c)

Figure $6 \mathrm{~b}$ Simulated precession diffraction pattern from $(8,0) \times 1$ ruck and tuck (c.f. the figure of ref (18) reproduced as Fig 6d)

Figure 6c Figure 3 of ref (17) reproduced with permission from Acta Crystallographica) 
Figure 6d The figure from ref (18) reproduced with permission from Acta Crystallographica)

\section{References:}

1. B. T. Kelly, W. H. Martin, A. M. Price, J. T. Bland, The mechanism of dimensional changes in the crystals of graphites and carbons under fast neutron irradiation. Philosophical Magazine 14, 343 (1966).

2. K. Urita, K. Suenaga, T. Sugai, H. Shinohara, S. lijima, In situ observation of thermal relaxation of interstitial-vacancy pair defects in a graphite gap. Physical Review Letters 94, 155502 (2005).

3. S. Muto, T. Tanabe, Damage process in electron-irradiated graphite studied by transmission electron microscopy. I. High-resolution observation of highly graphitized carbon fibre. Philosophical Magazine $A$ 76, 679 (1997).

4. A. Asthana et al., Investigations on the structural disordering of neutronirradiated highly oriented pyrolytic graphite Journal of Applied Crystallography 38, 361 (2005).

5. G. M. Jenkins, Deformation mechanisms in carbons. Chemistry and Physics of Carbon 11, 189 (1973).

6. R. H. Telling, M. I. Heggie, Radiation Damage in Graphite. Philosophical Magazine 87, 4796 (2007).

7. B. T. Kelly, The Physics of Graphite. (Applied Science, London, 1981).

8. L. Bochirol, E. Bonjour, Irradiations du graphite a basse temperature aux neutrons et aux electrons. Mesure de l'énergie émmagasiné. Carbon 6 , 661 (1966).

9. L. Arnold, Windscale 1957: Anatomy of a Nuclear Accident. (Palgrave Macmillan, London, 1995).

10. C. P. Ewels, R. H. Telling, A. A. El-Barbary, M. I. Heggie, P. R. Briddon, Metastable Frenkel pair defect in graphite: source of Wigner energy. Physical Review Letters 91, 025505 (2003).

11. P. A. Thrower, The study of defects in graphite by transmission electron microscopy. The Chemistry and Physics of Carbon, ed. P.L. Walker, Jr. (Marcel Dekker Inc: New York) 5, 217 (1969).

12. B. T. Kelly, The theory of irradiation damage in graphite. Carbon 15, 117 (1977).

13. L. M. Brown, A. Kelly, R. M. Mayer, The influence of boron on the clustering of radiation damage in graphite. II. Nucleation of interstitial loops. Philosophical Magazine 19, 721 (1969).

14. W. N. Reynolds, P. A. Thrower, Nucleation of Radiation Damage in Graphite. Philosophical Magazine 12, 573 (1965).

15. D. G. Martin, W. Henson, Scattering of long wavelength neutrons by defects in neutron irradiated graphite. Philosophical Magazine 9, 659 (1964).

16. C. D. Latham et al., The di-interstitial in graphite. Journal of Physics:

Condensed Matter 20, 395220 (2008). 
17. W. T. Eeles, Diffuse Diffraction Phenomena from Neutron-Irradiated Graphite Single Crystals. Acta Crystallographica A24, 688 (1968).

18. W. T. Eeles, Interpretation of the diffuse phenomena of neutron-irradiated graphite crystals. Philosophical Magazine 32, 1273 (1975).

19. M. I. Heggie et al., in Management of Ageing Processes in Nuclear Reactor Core G. B. Neighbour, Ed. (Royal Society of Chemistry, London, Cardiff, UK., 2007), pp. 83-90.

20. C. P. Ewels, M. I. Heggie, P. R. Briddon, Adatoms and the nanoengineering of carbon. Chemical Physics Letters 351, 178 (2002).

21. M. I. Heggie, I. Suárez-Martínez, G. Savini, G. L. Haffenden, J. M. Campanera, Radiation damage - a new model. International Atomic Energy Agency Technical Document 1647, (2010).

22. R. H. Telling, C. P. Ewels, A. A. El-Barbary, M. I. Heggie, Wigner defects bridge the graphite gap. Nature-Materials 2, 333 (2003).

23. R. H. Telling, M. I. Heggie, Stacking fault and dislocation glide on the basal plane of graphite. Philosophical Magazine Letters 83, 411 (2003).

24. D. Lexa, A. J. Kropf, Thermal, structural, and radiological properties of irradiated graphite from the ASTRA research reactor - Implications for disposal. Journal of Nuclear Materials 348, 122 (Jan, 2006).

25. S. K. Pregler, T. Hayakawa, H. Yasumatsu, T. Kondow, S. B. Sinnott, Combined computational and experimental study of Ar beam induced defect formation in graphite Nuclear Instruments and Methods in Physics Research Section B - Beam Interactions with Materials and Atoms $\mathbf{2 6 2}$, 240 (2007).

26. T. Iwata, T. Nihira, H. Matsuo, Irradiation and annealing effects on the caxis electrical resistivity of graphite. Journal of the Physical Society of Japan 36, 123 (1974).

27. I. L. Spain, Electronic transport properties of graphite, carbons, and related materials. Chemistry and Physics of Carbon 16, 119 (1981).

28. J. P. Hirth, A brief history of dislocation theory. Metallurgical Transactions A 16A, 2085 (1985).

29. P. A. Thrower, R. M. Mayer, Point defects and self-diffusion in graphite. Physica Status Solidi (a) 47, 11 (1978).

30. R. J. Price, J. C. Bokros, Annealing of neutron-irradiated pyrocarbons. Carbon 9, 555 (1971).

31. W. Bollman, Electron microscope of radiation damage in graphite. Journal of Applied Physics 32, 869 (1961).

32. A. Rappe, C. Casewitt, K. Colwell, W. Goddard-III, W. Skiff, UFF, a full periodic-table force-field for molecular mechanics and molecular dynamics simulations. Journal of the American Chemical Society 114, 10024 (1992).

33. G. E. Bacon, W. B.E., X-ray diffraction studies of neutron-irradiated graphite. Acta Crystallographica 9, 1029 (1956).

34. L. J. Teutonico, Moving edge dislocations in cubic and hexagonal materials. Physical Review 125, 1530 (1962). 
35. L. J. Teutonico, Dynamical behaviour of dislocations. Physical Review 124, 1039 (1961).

36. P. R. Briddon, R. Jones, LDA calculations using a basis of Gaussian orbitals. Physica Status Solidi B-Basic Research 217, 131 (Jan, 2000).

37. M. J. Rayson, P. R. Briddon, Rapid iterative method for electronicstructure eigenproblems using localised basis functions. Computer Phys. Comm/178, 128 (2008).

38. C. Hartwigsen, S. Goedecker, J. Hutter, Relativistic separable dual-space Gaussian pseudopotentials from H to Rn. Physical Review B 58, 3641 (1998).

39. H. Monkhorst, J. Pack, Special points for Brillouin-zone integrations. Physical Review B 13, 5188 (1976). 